Original article

\title{
Diversity of piroplasmids among wild and domestic mammals and ectoparasites in Pantanal wetland, Brazil
}

\author{
Keyla Carstens Marques de Sousa ${ }^{\mathrm{a}}$, Marina Pugnaghi Fernandes ${ }^{\mathrm{a}}$, Heitor Miraglia Herrera ${ }^{\mathrm{b}}$, \\ Carla Roberta Freschi ${ }^{\mathrm{c}}$, Rosangela Zacarias Machado ${ }^{\mathrm{a}}$, Marcos Rogério Andréa,*

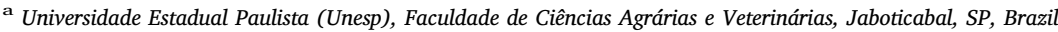 \\ b Universidade Católica Dom Bosco, Campo Grande, MS, Brazil \\ ${ }^{c}$ Imunodot Diagnósticos Ltda, Jaboticabal, SP, Brazil
}

\section{A R T I C L E I N F O}

\section{Keywords:}

Babesia

Cytauxzoon

Dogs

Phylogenetic analysis

Theileria

Rangelia

Wildlife

\begin{abstract}
A B S T R A C T
Piroplasmoses are one of the most prevalent arthropod-borne diseases of animals. The present work aimed to investigate the occurrence of piroplasmid in wild mammals, domestic dogs and ectoparasites in southern Pantanal region, central-western Brazil. For that purpose, blood or tissue samples from 31 Nasua nasua, 78 Cerdocyon thous, 7 Leopardus pardalis, 42 dogs, 110 wild rodents, and 30 marsupials, and 1582 ticks were submitted to PCR assays for piroplasmid targeting 18SrRNA and hps70 genes. Seven dogs, one C. thous, five $L$. pardalis, three N. nasua, six wild rodents, eight Amblyomma parvum, two Amblyomma sculptum and one Amblyomma ovale were positive for piroplasmid-PCR assays. Genotypes closely related to Babesia vogeli were detected in six dogs and five wild rodents. While genotypes closely related to Babesia caballi were detected in one C. thous, one $\operatorname{dog}$, one A. ovale and one A. sculptum, genotypes closely related to Babesia bigemina and Babesia bovis were detected in four A. parvum ticks. Four sequences obtained from A. parvum, three coatis and one wild rodent were closely related to Theileria equi. Cytauxzoon spp. was detected in four ocelots. The present study revealed that wild and domestic animals in Brazilian southern Pantanal are exposed to different piroplasmid species.
\end{abstract}

\section{Introduction}

Piroplasmid (Piroplasmida) are apicomplexan protozoa including the genera Babesia, Theileria, Cytauxzoon and Rangelia (Yabsley and Shock, 2013). These agents are tick-borne protozoans that parasitize blood cells of numerous wild and domestic vertebrates worldwide (Alvarado-Rybak et al., 2016). These parasites have a great economic and veterinary impact, being considered the second most commonly parasites found in the blood of mammals after trypanosomes (Schnittger et al., 2012). In the vertebrate hosts, the infection is usually characterized by fever, anemia and hemoglobinuria, and in severe cases, can lead to death (Kuttler, 1988). Although some of these parasites can cause diseases in animals and humans, the vectors are still unknown for many piroplasm species (Kjemtrup et al., 2000; Hersh et al., 2012).

Previously, the classification of piroplasmids relied only on host of origin, size and shape of trophozoites (small or large) and the number of merozoites within erythrocytes. However, the identification based on host origin has been invalidated, since many of these parasites are not host-specific (Penzhorn, 2006; Criado-Fornelio et al., 2003; Yabsley and Shock, 2013). Besides, the diagnosis based only on direct observations of blood smears does not always allow species identification and usually molecular assays are necessary in order to identify the etiological agent involved (Criado-Fornelio et al., 2003). In the last few years, the advent of molecular techniques has contributed to an expressive increase in the number of studies reporting infection with piroplasmids in wild animals worldwide (Alvarado-Rybak et al., 2016).

In Brazil, there are few reports concerning the seroprevalence and molecular detection of piroplasmid in wild carnivores. For instance, André et al. (2011) found a seroprevalence of $31.7 \%$ and $10.3 \%$ against $B$. vogeli antigen among wild felines and canids maintained in captivity, respectively. Additionally, André et al. (2011) detected a genotype closely related to $B$. leo in a neotropical wild cat (Oncifelis colocolo) and Cape genet (Genetta tigrina) also maintained in captivity in zoos in the state of São Paulo, Brazil. In addition to this, fatal cases of cytauxzoonosis were reported in two lions maintained in capitivity in a zoo in the

\footnotetext{
* Corresponding author at: Laboratório de Imunoparasitologia, Departamento de Patologia Veterinária, Faculdade de Ciências Agrárias e Veterinárias Júlio de Mesquita Filho (UNESP), Campus de Jaboticabal, Via de Acesso Prof. Paulo Donato Castellane, s/n, Zona Rural, CEP: 14884-900, Jaboticabal, São Paulo, Brazil.

E-mail address: marcos_andre@fcav.unesp.br (M.R. André).
} 


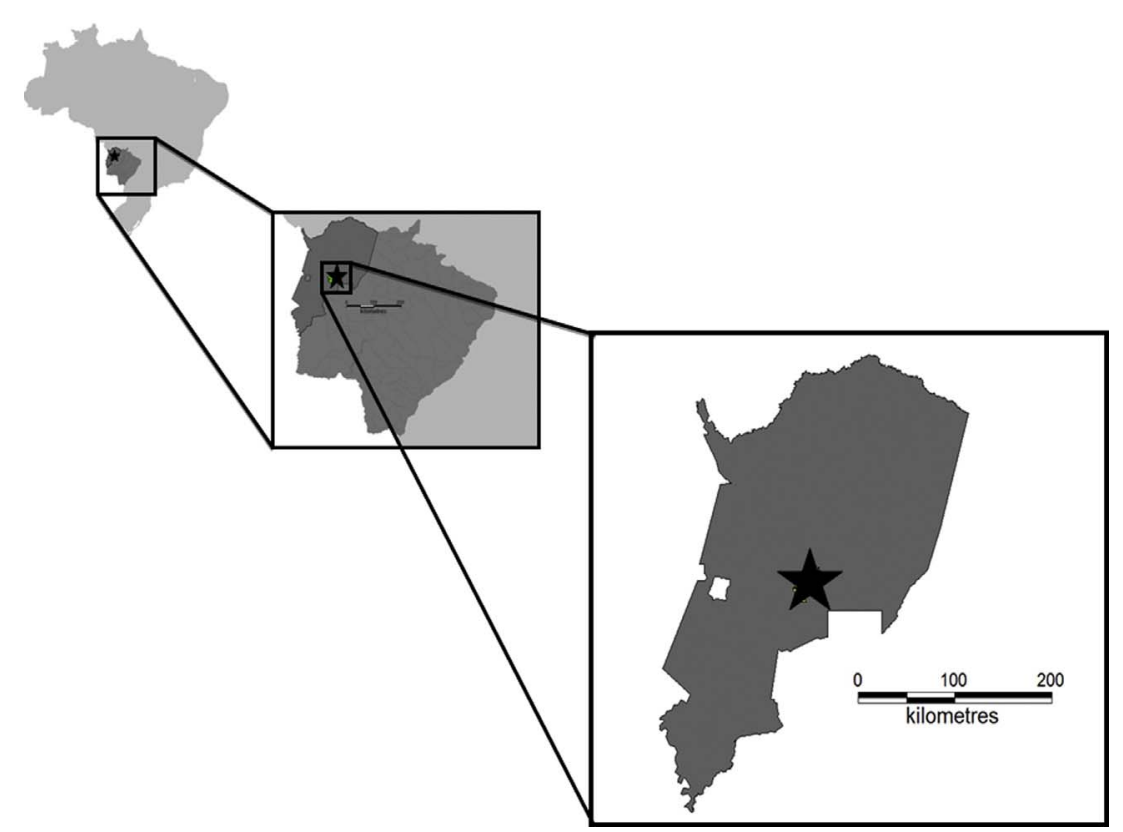

Fig. 1. Capture sites. Map of Mato Grosso do Sul State, central-western Brazil, showing the Pantanal region, where mammals' blood and spleen samples and ticks were collected in the present study. state of Rio de Janeiro (Peixoto et al., 2007). Besides, André et al. (2009) detected Cytauxzoon DNA closely related to Cytauxzoon felis in asymptomatic neotropical felines also maintained in captivity in zoos in the cities of São Paulo and Brasília. Furthermore, Cytauxzoon sp. has also been molecularly detected in domestic cats from the states of Rio de Janeiro (Maia et al., 2013) and Mato Grosso do Sul (André et al., 2015).

Due to the lack of information about the epidemiology and transmission routes of piroplasms among wild animals in Brazil, the present study aimed to investigate the occurrence of piroplasmids in wild mammals and domestic dogs and their respective ectoparasites in the region of Pantanal, state of Mato Grosso do Sul, central-western Brazil.

\section{Materials and methods}

\subsection{Study area}

The fieldwork was conducted at the Nhumirim ranch $\left(56^{\circ} 39^{\prime} \mathrm{W}\right.$, $18^{\circ} 59^{\prime} \mathrm{S}$ ), located in the central region of the Pantanal, municipality of Corumbá, state of Mato Grosso do Sul, central-western Brazil (Fig. 1). This region is characterized by a mosaic of semi-deciduous forest, arboreal savannas, seasonally flooded fields covered by grasslands with dispersed shrubs and several temporary and permanent ponds. The Pantanal is the largest Neotropical floodplain, being well known for its rich biodiversity. Two well-defined seasons are recognized in that region: a rainy summer (October to March) and a dry winter (April to September) (Sousa et al., 2017a, 2017b).

\subsection{Biological sampling}

Between August 2013 and March 2015, a total of 256 animals were captured in the central region of the Pantanal, municipality of Corumbá, state of Mato Grosso do Sul: 158 carnivores, among them 78 crab-eating foxes (C. thous), 31 coatis ( $N$. nasua) and seven ocelots ( $L$. pardalis); 140 small mammals, among them 110 wild rodents (77 Thrichomys fosteri, 25 Oecomys mamorae and 8 Clyomys laticeps) and 30 wild marsupials (14 Thylamys macrurus, 11 Gracilinanus agilis, 4 Monodelphis domestica and 1 Didelphis albiventris). Additionally, 42 blood samples from domestic dogs cohabiting the same studied area were collected. Blood samples were collected from carnivores and domestic dogs by puncture of the cephalic vein and stored in Vacutainer tubes with EDTA and without EDTA, in order to obtain total blood and serum samples for molecular and serological assays, respectively. Spleen samples were collected from small mammals and stored in absolute ethanol (Merck", Kenilworth, Nova Jersey, USA) for molecular assays. All blood and serum samples were stored at $-20{ }^{\circ} \mathrm{C}$. The DNA extraction and serological assays were performed one week after the captures. All animal captures were in accordance with the licenses obtained from the Brazilian Government Institute for Wildlife and Natural Resources Care (IBAMA) (license numbers 38145, 38787-2) and endorsed by the Ethics Committee of FCAV/UNESP University (Faculdade de Ciências Agrárias e Veterinárias, Universidade Estadual Paulista "Júlio de Mesquita Filho", Câmpus Jaboticabal) n 006772/13 (Sousa et al., 2017a, 2017b).

One thousand five hundred and eighty-two ticks parasitizing the sampled mammals were collected, of which 1033 (65.2\% [115 adults and 918 nymphs]) belonging to A. sculptum Berlese, 241 (15.2\% [78 adults and 163 nymphs]) belonging to $A$. parvum Aragão, 32 (2\%) $A$. ovale Koch adults, one (0.06\%) Amblyomma tigrinum Koch adult, one (0.06\%) Rhipicephalus (Boophilus) microplus (Canestrini) adult, one (0.06\%) Rhipicephalus sanguineus s.l. (Latreille) adult, four $(0.2 \%)$ Amblyomma auricularium (Conil) nymphs, and 269 (17\%) Amblyomma larvae. Besides, a total of 80 Polygenis (Polygenis) bohlsi bohlsi (Wagner) fleas were collected (Sousa et al., 2017a, 2017b).

\subsection{Giemsa-stained blood smears}

Blood smears were performed using peripheral blood collected from wild carnivores and domestic dogs, fixed with methanol and stained with Giemsa (Giemsa stain, modified, Sigma-Aldrich ${ }^{\circledR}$, St. Louis, MO, USA).

\subsection{Enzyme-linked immunosorbent assay (ELISA)}

In order to detect IgG antibodies to B. vogeli, canids ( $C$. thous and dogs) serum samples were individually tested by an ELISA assay using a commercial kit (IMUNODOT, Diagnósticos Ltda", Jaboticabal, SP, Brazil), according to the manufacturer's instructions.

\subsection{DNA extraction}

DNA was extracted from $200 \mu \mathrm{L}$ of each whole blood (158 wild 
Table 1

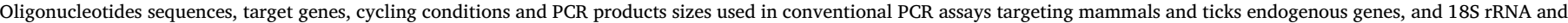
heat shock protein 70 ( $h s p 70$ ) piroplasmid genes in biological samples from wild mammals domestic dogs and their respective ectoparasites, sampled in Pantanal wetland, Brazil.

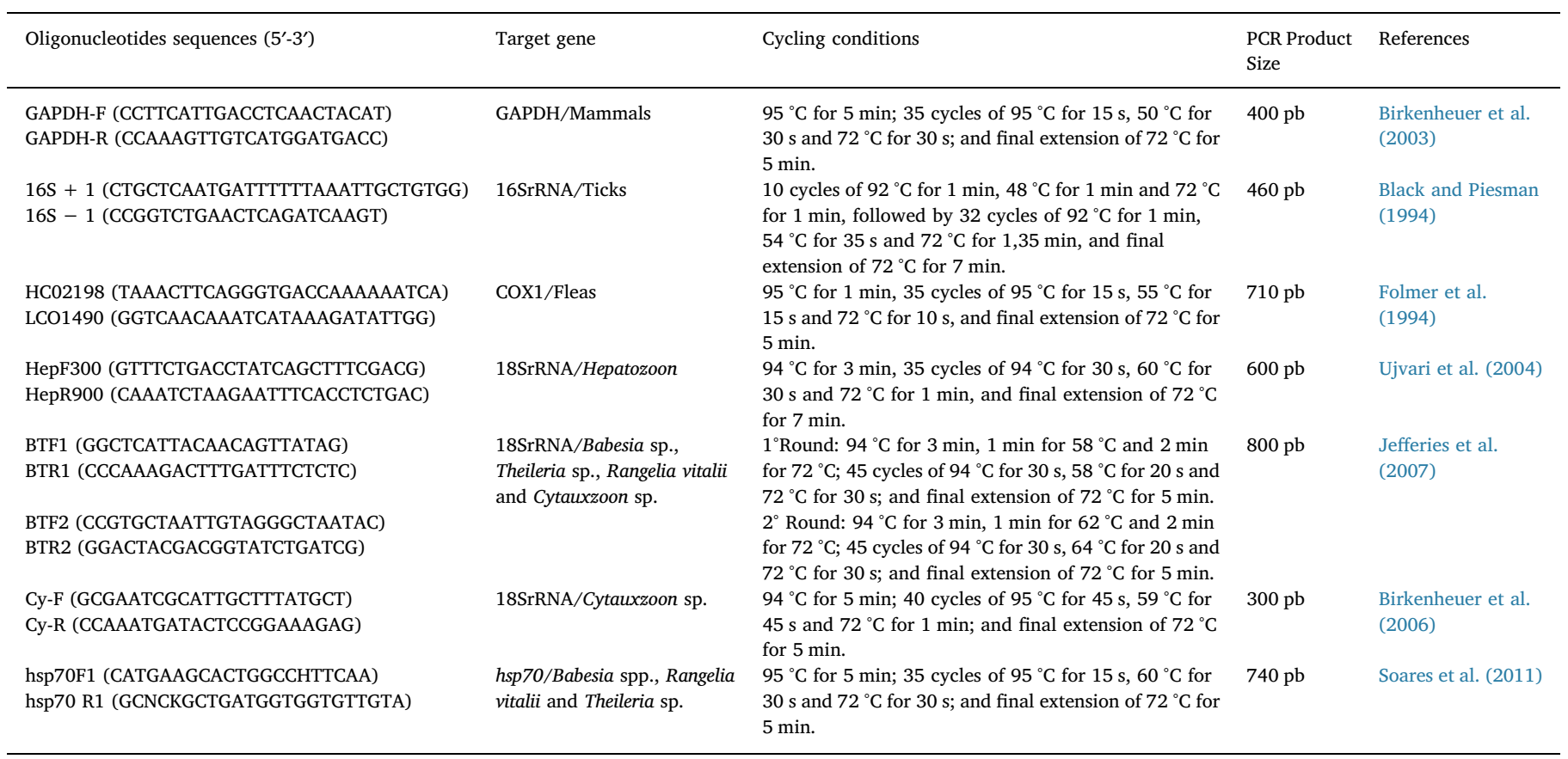

carnivores and 42 domestic dogs) and $10 \mathrm{mg}$ of spleen (140 small mammals) samples using the QIAamp DNA Blood Mini kit (QIAGEN ${ }^{\circ}$, Valencia, CA, USA), according to the manufacturer's instructions. The total number of individual tick samples or pools was 523, of which 228 (43.5\%) were from adults, 256 (48.9\%) pooled nymphs, and $39(7.4 \%)$ from pooled larvae. DNA extraction from ticks was processed in pools for nymphs (up to 5 individuals) and larvae (up to 10 individuals), while the adults were processed individually. A total of 39 pooled fleas samples were submitted to DNA extraction. DNA extraction from fleas was also performed in pools consisting of up to five individuals. Ticks and fleas were macerated and submitted to DNA extraction, using the same kit before mentioned (Sousa et al., 2017a, 2017b).

\subsection{PCR for endogenous genes}

In order to verify the presence of amplifiable DNA in the samples, internal control PCR assays targeting fragments of mammalian glyceraldehyde-3-phosphatedehydrogenase (GAPDH), ticks mitochondrial 16S rRNA and fleas cytochrome-c oxidase subunit I (COX1) genes were performed (Table 1). All 298 DNA animal samples amplified the predicted product for GAPDH gene. Out of 523 sampled ticks, 31 (5.9\% [23 A. parvum adults, 4 A. sculptum adults, 1 A. ovale adult, 1 A. parvum nymph and 2 pooled Amblyomma larvae]) showed negative results for the tick mitochondrial 16S rRNA gene and were excluded from subsequent analyses. Only one flea DNA sample did not amplify the predicted product for cox-1 and was also excluded from subsequent analyses (Sousa et al., 2017a, 2017b).

\subsection{PCR assays}

Previously described PCR protocols based on 18SrRNA gene and heat shock protein 70 gene (hsp70) were performed in order to amplify Babesia spp., Cytauxzoon sp. and Theileria sp. DNA (Ujvari et al., 2004; Birkenheuer et al., 2006; Jefferies et al., 2007; Soares et al., 2011) (Table 1). Each sample of extracted DNA was used as a template in $25 \mu \mathrm{L}$ PCR reactions. The mixture containing 10X PCR buffer (Life Technologies ${ }^{\circledR}$, Carlsbad, CA, USA), $1.0 \mathrm{mM} \mathrm{MgCl}_{2}$ (Life Technologies',
Carlsbad, CA, USA), $0.2 \mathrm{mM}$ deoxynucleotide triphosphate (dNTPs) mixture (Life Technologies ${ }^{\circ}$ Carlsbad, CA, USA), 1.5 U Taq DNA Polymerase (Life Technologies, Carlsbad, CA, USA), and $0.5 \mu \mathrm{M}$ of each primer (Integrated DNA Technologies ${ }^{\circ}$, Coralville, IA, USA). Babesia vogeli, Cytauxzoon sp. and Theileria sp. DNA positive controls were obtained from naturally infected cats (André et al., 2015). Ultra-pure sterile water (Life Technologies ", Carlsbad, CA, USA) was used as negative control. PCR products were separated by electrophoresis on $1 \%$ agarose gel stained with ethidium bromide (Life Technologies, Carlsbad, CA, USA). In order to prevent PCR contamination, DNA extraction, reaction setup, PCR amplification and electrophoresis were performed in separated rooms. The gels were imaged under ultraviolet light using the Image Lab Software version 4.1 (Bio-Rad $\left.{ }^{\circ}\right)$. The reaction products were purified using the Silica Bead DNA gel extraction kit (Thermo Fisher Scientific ${ }^{\circ}$, Waltham, MA, USA). The sequencing was carried out using the BigDye Terminator v3.1Cycle Sequencing Kit (Thermo Fisher Scientific ${ }^{\circ}$, Waltham, MA, USA) and ABI PRISM 310DNA Analyzer (Applied Biosystems ${ }^{\circ}$, Foster City, CA, EUA) (Sanger et al., 1977).

\subsection{Bioinformatics/Phylogenetic analysis}

The sequences obtained from positive samples were first submitted to a screening test using Phred-Phrap software version 23 (Ewing and Green, 1998; Ewing et al., 1998) in order to evaluate the electropherogram quality and obtain consensus sequences from the alignment of sense and antisense sequences. The BLAST program (Altschul et al., 1990) was used to analyze the sequences of nucleotides (BLASTn), aiming to browse and compare with sequences from international database (GenBank) (Benson et al., 2002). All sequences that showed appropriate quality standards and identity with piroplasmid species were deposited in GenBank. Samples showing positive results for both PCR protocols (18SrRNA and $h s p 70$ ) had their sequences concatenated, using the Fragment Merger software version 1 (Bell and Kramvis, 2013). The sequences were aligned with sequences published in GenBank using MAFFT software, version 7 (Katoh and Standley, 2013).

Phylogenetic inference was based on Bayesian Inference (BI). The BI 
analysis was performed with MrBayes 3.1.2 (Ronquist and Huelsenbeck, 2003). Markov chain Monte Carlo (MCMC) simulations were run for $10^{9}$ generations with a sampling frequency of every 100 generations and a burn-in of $25 \%$. The best model of evolution was selected by the program jModelTest 2 (version 2.1.6) on XSEDE (Darriba et al., 2012), under the Akaike Information Criterion (AIC) (Posada and Buckley, 2004). All phylogenetic analyses were performed using CIPRES Science Gateway (Miller et al., 2010). The trees were examined in Treegraph 2.0.56-381 beta (Stover and Muller, 2010).

\section{Results}

The average absorbance of negative sera provided in ELISA kit was $0.157(0.198 \pm 0.129)$, resulting in a cut-off value of 0.393 . Thirtynine $(92.8 \%)$ dogs and $42(53.8 \%) C$. thous were seroreactive to $B$. vogeli antigen. The mean antibodies absorbance values of seropositive dogs and crab-eating foxes were $0.734(1.431 \pm 0.450)$ and 0.691 $(1.211 \pm 0.411)$, respectively.

Seven $(16.6 \%)$ dogs, one (1.2\%) crab-eating fox, three $(42.8 \%)$ ocelots, six (7.8\%) T. fosteri wild rodents, eight (6\%) A. parvum (5 adults and 3 nymphal pools), two (0.6\%) A. sculptum (1 adults and 1 nymphal pool) and one (3.1\%) A. ovale adult (1.2\%) were positive for 18SrRNAPCR based on Jefferies et al. (2007) protocol. Three (9.6\%) coatis and one (14.2\%) ocelot were positive for 18SrRNA-PCR based on Ujvari et al. (2004) and Birkenheuer et al. (2006) protocols, respectively. Three out of seven positive dogs in 18SrRNA-PCR and two out of eight positive A. parvum in 18SrRNA-PCR were also positive in hsp70-PCR based on Soares et al. (2011) protocol. All the sampled fleas were negative in PCRs assays for piroplasmids. All sequences obtained from the positive samples were deposited in GenBank international database under the accession numbers KY450716-KY450752.

Regarding the presence of inclusions suggestive of piroplasmid in Giemsa-stained blood smears, piroplasms and Maltese cross forms were observed within one ocelots erythrocytes. Additionally, piroplasms were found in one coatís erythrocytes (Fig. 2). Both animals were positive in 18SrRNA PCR assays for piroplasmid.

Six out of seven $18 \mathrm{~S}$ rRNA-piroplasmid sequences obtained from domestic dogs showed $100 \%$ of identity with $B$. vogeli previously deposited in GenBank (KT323934) by BLAST analysis. One 18S rRNApiroplasmid sequence obtained from a domestic dog showed $100 \%$ of identity with B. caballi (EU642512). While five out of six 18S rRNApiroplasmid sequences obtained from T. fosteri showed 99\% of identity with B. vogeli (KT323934), one of them showed $99 \%$ of identity with $T$. equi (KU672386) by BLAST analysis. One 18S rRNA-piroplasmid sequence obtained from a crab-eating fox, one sequence obtained from an $A$. ovale adult and one sequence obtained from an A. sculptum adult showed $100 \%$ of identity with $B$. caballi previously deposited in GenBank (EU642512). While four 18S rRNA-piroplasmid sequences obtained from A. parvum adults showed $99 \%$ of identity with $T$. equi (KU672386), four others 18S rRNA-piroplasmid sequences obtained from A. parvum (1 adult and three nymphal pools) showed $97 \%$ of identity with a sequence of Babesia sp. obtained from Odocoileus virginianus, from USA (HQ264119). An 18S rRNA-piroplasmid sequence obtained from an A. sculptum nymphal pool showed $99 \%$ of identity with a sequence of Theileria sp. obtained from Rangifer tarandus tarandus, also from USA (JN086224).

Surprisingly, three coatis' blood samples amplified piroplasmid DNA sequences using a PCR protocol for Hepatozoon based on 18SrRNA gene (Ujvari et al., 2004). The amplified sequences showed 100\% of identity with a Theileria sp. sequence obtained from a domestic cat sampled in Brazil (KF970930). These results were obtained in a previous study that aimed to perform the molecular detection of Hepatozoon spp. in the same animal samples (Sousa et al., 2017a). These results showed that the primers previously designed by Ujvari et al. (2004) may also anneal to piroplasmid DNA.

All 18S rRNA-piroplasmid sequences obtained from ocelots $(n=4)$ showed $99 \%$ of identity with C. felis previously deposited in GenBank (GU903911) by BLAST analysis (Tables 2 and 3). Three hsp70-piroplasmid sequences obtained from domestic dogs showed $100 \%$ of identity with B. vogeli (AB248733). Finally, two hsp70-piroplasmid sequences obtained from A. parvum nymphal pools showed $90 \%$ of identity with $B$. canis (AB248735), by BLAST analysis.

The phylogenetic analysis based on 18S rRNA gene fragments clustered six piroplasmid sequences obtained from dogs' blood samples (KY450732, KY450733, KY450734, KY450735, KY450736, KY450737) and five sequences obtained from T. fosteri spleen samples (KY450738,

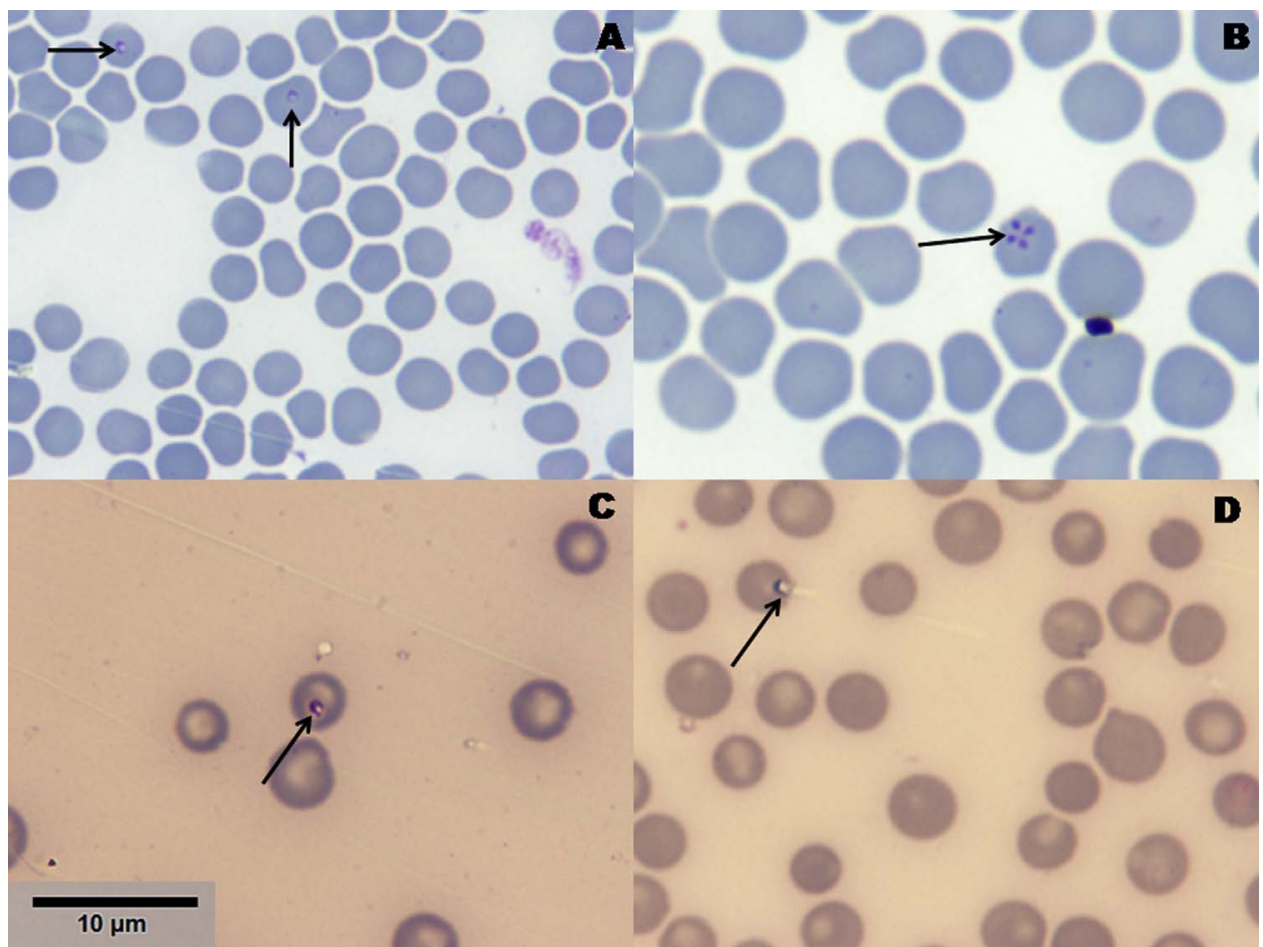

Fig. 2. Forms morphologically similar to piroplasmids found in Giemsa-stained blood smears from sampled mammals in Brazilian southern Pantanal. (A) Individual forms found within erythrocytes from an ocelot. (B) Maltese-cross inclusion found within erythrocytes from an ocelot. (C; D) Individual forms found within erythrocytes from coatis sampled in southern Pantanal. 
Table 2

Maximum identity of $18 \mathrm{~S}$ rRNA and $h s p 70$ piroplasmid sequences detected in wild and domestic animals in Brazilian Pantanal by BLAST analysis.

\begin{tabular}{|c|c|c|c|}
\hline Host & Number of sequences analyzed & Target gene & $\%$ identity by BLAST ${ }^{*}$ - analysis \\
\hline Cerdocyon thous & 1 & 18SrRNA & $100 \%$ Babesia caballi genotype A_CABEQ30 (EU642512) \\
\hline Thrichomys fosteri & 5 & 18SrRNA & 99\% Babesia vogeli (KT323934) \\
\hline Thrichomys fosteri & 1 & 18SrRNA & 99\%Theileria equi (KU672386) \\
\hline Canis familiaris & 5 & 18SrRNA & $100 \%$ Babesia vogeli (KT323934) \\
\hline Canis familiaris & 1 & 18SrRNA & $100 \%$ Babesia caballi genotype A_CABEQ30 (EU642512) \\
\hline Nasua nasua & 3 & 18SrRNA & 100\% Theileria sp. (KP410272) \\
\hline Leopardus pardalis & 4 & 18SrRNA & 99\% Cytauxzoon felis (GU903911) \\
\hline Canis familiaris & 3 & hsp70 & $100 \%$ Babesia vogeli (AB248733) \\
\hline
\end{tabular}

KY450739, KY450740, KY450741, KY450742) in a branch comprising B. vogeli sequences (AY371196, HM590440) previously deposited in GenBank, with clade support value of 100 , based on BI analysis. 18SrRNA-piroplasmid sequences obtained from one C. thous blood sample (KY450725), one domestic dog blood sample (KY450731), one A. ovale adult (KY450726), and one A. sculptum adult (KY450730) were closely related to B. caballi (EU888901, EU642512), with clade support value of 100, based on BI. Four 18SrRNA-piroplasmid sequences obtained from A. parvum (1 adult [KY450743], three sequences obtained from nymphal pools [KY450727, KY450728, KY450729]), and a Babesia sp. sequence, previously deposited in GenBank and obtained from Odocoileus virginianus sampled in the USA (HQ264119), were grouped in the same clade of $B$. bigemina (JQ723014) and $B$ bovis (L19077) based on BI analysis. 18SrRNA-piroplasmid sequences obtained from four A. parvum adults (KY450716, KY450717, KY450718, KY450716), three coatis blood samples (KY450722, KY450723, KY450724) and one T. fosteri spleen sample (KY450720) were grouped into the same large branch of T. equi sequences (KU672386, AY150064, AB515315), with clade support value of 99 in BI analysis. One 18SrRNA-piroplasmid sequence obtained from an A. sculptum nymphal pool (KY450721) was placed in the same clade of Theileria sp. sequence obtained from Rangifer tarandus tarandus (JN086224) sampled in the USA, with clade support value of 100 , based on BI analysis. Four 18SrRNA-piroplasmid sequences obtained from ocelots blood samples (KY450744, KY450745, KY450746, KY450747) was pooled in the same clade of $C$. felis sequences previously deposited in GenBank (DQ382277, L19080), with clade support value of 100, based on BI analysis (Fig. 3). 18SrRNA-phylogenetic inference based on BI method was performed using the evolutionary model GTR $+\mathrm{G}+\mathrm{I}$ and Coccidia sp. (HM117907), Isospora suis (U97523), Sarcocystis sp. (U97524), and Adelina bambarooniae (AF494059) as outgroups.

Regarding the concatenated phylogenetic analysis of piroplasmid based on 18SrRNA and $h s p 70$ genes fragments, two piroplasms sequences obtained from $A$. parvum nymphal pools were pooled in the same branch of $R$. vitalii sequences (JF279603; KF218606), with clade support value of 94 , based on BI analysis. The remaining three piroplasms $h s p 70+18$ SrRNA concatenated sequences obtained from dogs blood samples were grouped in the same clade of $B$. vogeli sequences (AB248733; AY371196), with clade support value of 100 in BI analysis (Fig. 4). Concatenated 18SrRNA + hsp70-phylogenetic inference based on BI method were performed using the GTR + G + I evolution model and Plasmodium falciparum (M19753; JQ627152) as outgroup.

\section{Discussion}

The present study showed the presence of piroplasmid DNA in blood or spleen samples from wild carnivores, domestic dogs, rodents and ticks sampled in the southern region of Pantanal, state of Mato Grosso do Sul, central-western Brazil. Molecular analyses based on 18S rRNA and $h s p 70$ genes revealed an occurrence of different piroplasmid species among sampled mammals and arthropods.

Babesia vogeli is a widespread tick-borne protozoan in dogs in Brazil (Passos et al., 2005; O’Dwyer et al., 2009; Sousa et al., 2013; da Costa et al., 2015; Moraes et al., 2015), in accordance with the wide distribution of its vector, $R$. sanguineus s.l., mainly in urban and peripheral urban areas (Labruna and Pereira, 2001). In the present study, the molecular occurrence of $B$. vogeli (16.6\%) among sampled dogs was higher than that found (3.3\%) in a previous study involving dogs in an urban area from the same state of Mato Grosso do Sul (Sousa et al., 2013) and dogs (3.13\%) sampled in northern Pantanal, state of Mato Grosso (Melo et al., 2016). Herein, the high seroprevalence (92.8\%) to B. vogeli found among dogs was similar to that found (81.6\%) among dogs sampled in an urban area from the same state of Mato Grosso do Sul (Sousa et al., 2013). Infection by B. vogeli in dogs seems to be common among dogs from the state of Mato Grosso do Sul.

On the other hand, reports of seropositivity to B. vogeli among wild canids are scarce in Brazil (André et al., 2011). A higher seroprevalence (53.8\%) was found among free-living crab-eating foxes sampled in the present study when compared to that found among with wild canids (10\%) maintained in captivity in Brazilian zoos (André et al., 2011). Although this work showed the first serological evidence of exposure to

Table 3

Maximum identity of $18 \mathrm{~S}$ rRNA and hsp70 piroplasmid sequences detected in ticks collected from wild animals in Brazilian Pantanal by BLAST analysis.

\begin{tabular}{|c|c|c|c|}
\hline Tick specimen & Host & Target gene & $\%$ identity by BLAST ${ }^{\circ}$ analysis \\
\hline Amblyoma parvum adult & Cerdocyon thous & 18SrRNA & 99\%Theileria equi (KU672386) \\
\hline Amblyoma parvum adult & Cerdocyon thous & 18SrRNA & 99\%Theileria equi (KU672386) \\
\hline Amblyoma parvum adult & Cerdocyon thous & 18SrRNA & 99\%Theileria equi (KU672386) \\
\hline Amblyoma parvum adult & Cerdocyon thous & 18SrRNA & 99\%Theileria equi (KU672386) \\
\hline Amblyoma parvum adult & Cerdocyon thous & 18SrRNA & 97\% Babesia sp. from Odocoileus virginianus (HQ264119) \\
\hline Amblyoma parvum nymph & Thrichomys fosteri & 18SrRNA & 97\% Babesia sp. from Odocoileus virginianus (HQ264119) \\
\hline Amblyoma parvum nymph & Thrichomys fosteri & 18SrRNA & 97\% Babesia sp. from Odocoileus virginianus (HQ264119) \\
\hline Amblyoma parvum nymph & Clyomys laticeps & 18SrRNA & 97\% Babesia sp. from Odocoileus virginianus (HQ264119) \\
\hline Amblyomma sculptum adult & Cerdocyon thous & 18SrRNA & 100\% Babesia caballi genotype A_CABEQ30 (EU642512) \\
\hline Amblyomma sculptum nymph & Cerdocyon thous & 18SrRNA & 99\% Theileria sp. from Rangifer tarandus tarandus (JN086224) \\
\hline Amblyomma ovale adult & Cerdocyon thous & 18SrRNA & 100\% Babesia caballi genotype A_CABEQ30 (EU642512) \\
\hline Amblyoma parvum nymph & Thrichomys fosteri & hsp70 & 90\% Babesia canis (AB248735) \\
\hline Amblyoma parvum nymph & Clyomys laticeps & hsp70 & 90\% Babesia canis (AB248735) \\
\hline
\end{tabular}




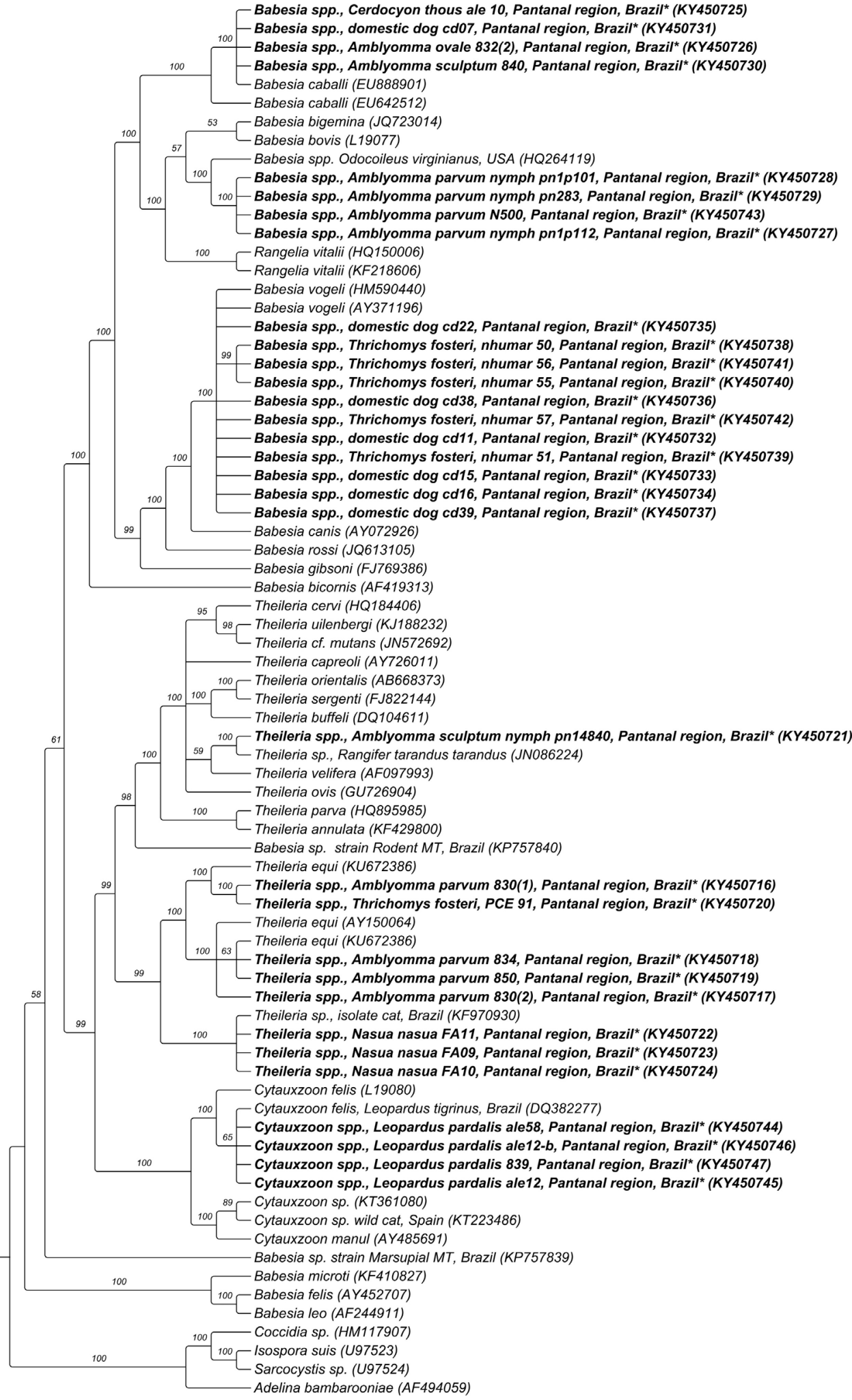

$\frac{1}{0.0} 0.04$

B. vogeli among free-living wild canids in Brazil, cross-reactions between Babesia and Rangelia could not be discarded. Furthermore, considering the high occurrence of $B$. vogeli found in domestic dogs sampled in the studied area, the role of these animals as a source of $B$. vogeli infection to wild carnivores cohabiting the same area should be better investigated.
Fig. 3. Phylogenetic tree constructed with $800 \mathrm{pb}$ of piroplasmid-18SrRNA sequences, using Bayesian method and GTR + G + I evolutionary model. Numbers at nodes correspond to Bayesian posterior probabilities over 50, using Coccidia sp. (HM117907), Isospora suis (U97523), Sarcocystis sp. (U97524), and Adelina bambarooniae (AF494059) as outgroups. 


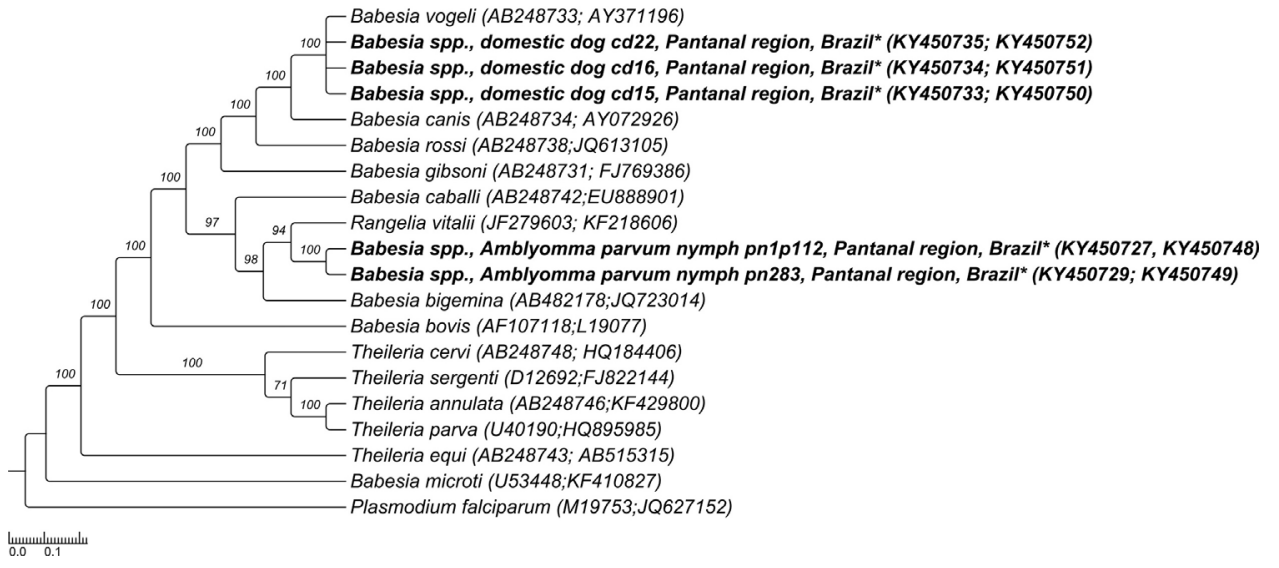

Fig. 4. Phylogenetic tree constructed with $1560 \mathrm{pb}$ of piroplasmid 18SrRNA + hsp70 sequences, using Bayesian method and GTR + G + I evolutionary model. Numbers at nodes correspond to Bayesian posterior probabilities over 50. Plasmodium falciparum (M19753; JQ627152) was used as outgroup. hosts has already been reported. For instance, while Theileria annulata, T. equi and B. caballi DNA were detected in blood samples from dogs in Croatia (Beck et al., 2009), B. canis and B. rossi DNA were detected in blood samples from horses in France (Fritz, 2010). Besides, genotypes closely related to $T$. equi have also been detected in cats in Brazil (André et al., 2014; 2015). These unusual findings suggest that some piroplasm species may show a lack of host specificity. Alternatively, new piroplasm species closely related to those already described in domestic animals may circulate in different hosts. Furthermore, the small gene fragments used in the present study may not have allowed a robust phylogenetic positioning of the amplified sequences.

Although Anocentor nitens (Roby and Anthony, 1963) and R. (B.) microplus (Battsetseg et al., 2002) ticks are considered, respectively, the main vectors for $B$. caballi and T. equi in Brazil, some evidence has suggested the participation of Amblyomma cajennense sensu lato ticks in T. equi (Kerber et al., 2009; Ribeiro et al., 2011; Scoles and Ueti, 2013) and $B$. caballi (Vieira et al., 2013) transmission cycles. Herein, while $B$. caballi DNA was detected in A. ovale and A. sculptum ticks, T. equi DNA was detected in $A$. parvum ticks. These findings highlight the importance of future studies regarding the role of Amblyomma species in the epidemiological cycles of $B$. caballi and $T$. equi.

In South America, Rangelia vitalii, a piroplasm species associated with hemolytic disorders (Soares et al., 2011) in canids, has been reported in domestic and wild canids from southeastern and southern regions of Brazil (Soares et al., 2011, 2014; Gottlieb et al., 2016; Quadros et al., 2015; Fredo et al., 2015; Silveira et al., 2016), and in domestic dogs from Argentina (Eiras et al., 2014) and Uruguay (Soares et al., 2015). Herein, the concatenated phylogenetic analysis revealed the presence of a genotype closely related to $R$. vitalii in A. parvum ticks collected from $T$. fosteri and $C$. laticeps wild rodents. Previous studies suggested that $C$. thous is the natural reservoir for $R$. vitalii in Brazil (Soares et al., 2014; Fredo et al., 2015). Considering the fact that $C$. thous is frequent found in Pantanal (Mamede and Alho, 2006), future studies should be performed in order to assess the real significance of the circulation of this genotype in this Brazilian wetland.

A genotype closely related to Theileria sp. previously detected in a wild deer ( $R$. t. tarandus) sampled in the USA was detected in A. sculptum nymphs collected from $C$. thous in the present study. Previously, a genotype showing $90-100 \%$ identicalness to Theileria cervi was detected in $52 \%$ of sampled pampas deer (Ozotoceros bezoarticus) in the same region of Pantanal (Silveira et al., 2013). Genotypes closely related to Theileria sp. associated to wild ruminants have also been detected in cats from Campo Grande, an urban area in the state of Mato Grosso do Sul (André et al., 2015). Future studies aiming at investigate the host and vectors range of wild ruminants-associated Theileria genotypes should be investigated in Brazilian Pantanal.

While the epidemiology of Cytauxzoon sp. has been extensively studied in the USA (Blouin et al., 1984; Meinkoth and Kocan, 2005; Yabsley et al., 2006; Haber et al., 2007), the occurrence of these parasites in other regions of the world has been scarcely reported. In fact, while clinical and fatal cytauxzoonosis has been well reported in the USA (Garner et al., 1996; Nietfield and Pollock, 2002), Germany (Jakob and Wesemeier, 1996) and Italy (Carli et al., 2012), the parasite has been rarely described and not associated with clinical disease in domestic cats in Brazil so far (Maia et al., 2013; André et al., 2015). Although Cytauxzoon sp. have already been reported in neotropical and exotic wild felids maintained in captivity in zoos in Brazil (Peixoto et al., 2007; André et al., 2009), the present work showed the first molecular detection of Cytauxzoon sp. among free-living wild felids in South America. Herein, a genotype closely related to $C$. felis was detected in four apparently asymptomatic ocelots. Cytauxzoon-suggestive maltese cross inclusions were found in one ocelot blood smear. The pathogenic potential of Cytauxzoon isolates in Brazil is still unknown. In fact, while fatal cytauxzoonosis has been reported in lions maintained in captivity in a zoo in the state of Rio de Janeiro (Peixoto et al., 2007), the parasite has been detected in apparently asymptomatic wild felids maintained in captivity in the state of São Paulo and Brasília (André et al., 2009). The role of neotropical wild felids as reservoirs for $C y$ tauxzoon in Brazil should be further investigated. Although A. parvum ticks were found parasitizing the sampled ocelots, Cytauxzoon DNA was not detected in any collected tick specimen. While Dermacentor variabilis (Blouin et al., 1984) and A. americanum (Reichard et al., 2010) are responsible for transmitting $C$. felis among felids in the USA, the vectors involved in the Cytauxzoon epidemiological cycles in Brazil remains unknown.

A genotype closely related to $B$. vogeli was also detected in five $T$. fosteri wild rodents. It seems that the host range for $B$. vogeli or a genotype closed related to is wider than previously supposed, since the protozoa has also been detected in cats (et al., 2014, 2015; et al., 2014, 2015), besides dogs and rodents, in Brazil. Changes in land use may facilitate the circulation of piroplasmid-infected ticks between wild rodents and domestic and wild animals, since those animals can share the same ecotypes. Recently, a genotype closely related to Theileria bicornis was detected, for the first time in the American continent, in $T$. fosteri rodents sampled in northern Pantanal (Wolf et al., 2016). Infections by T. bicornis have been reported only in black (Diceros bicornis michaeli) and white (Ceratotherium simum simum) rhinoceros from Africa (Otiende et al., 2015). The molecular detection of a genotype closely related to T. bicornis in America (Wolf et al., 2016) also represents an unusual finding. Indeed, the molecular analysis of a small subunit of 18SrRNA gene $(349 \mathrm{pb})$ might not have been sufficiently robust to allow an accurate phylogenetic positioning of the piroplasmid species detected in wild rodents from northern Pantanal. Future molecular studies targeting different genes are much needed in order to access the real identity of the piroplasm species circulating among Brazilian wild rodents.

Molecular studies aiming at identifying the phylogenetic positioning of piroplasmids have been mainly based on the small subunit ribosomal 
$18 \mathrm{~S}$ gene, due to its conserved nature, although others genes, such as cytochrome $\mathrm{b}$ and the heat-shock proteins encoding genes, have also been used (Irwin, 2009). However, a common problem observed when the molecular analyses are only based on 18S rRNA sequences is that the molecular findings usually do not reflect the piroplasm morphology (Allsopp et al., 1994; Schnittger et al., 2012). This discrepancy between molecular data and parasite morphology may be due to the complexity of $18 \mathrm{~S}$ secondary structure that could lead to inconsistencies in gene alignment (Morrison and Ellis, 1997). Mitochondrial genome sequences and structures have been showed as an alternative to analyze the relationships and for delineating specimens to the species level (Schreeg et al., 2016).

Recently, phylogenetic analyses based on mitochondrial genome sequences concatenated along with $18 \mathrm{~S}$ rRNA sequences identified five distinct Piroplasmida lineages with different biological features, namely: i. Babesia sensu stricto (B. caballi, B. bigemina, B. canis, B. rossi, B. vogeli, B. gibsoni and B. bovis); ii. Theileria sp. and Cytauxzoon sp.; iii. T. equi; iv. Western Babesia group (B. conradae); v. B. microti group (Schreeg et al., 2016). Although the mitochondrial genome analysis was not performed in the present study, sequences apparently belonging to three out of five Piroplasmida lineages were identified. Genotypes closely related to $B$. caballi and $B$. vogeli, obtained from $C$. thous, T. fosteri, A. ovale and A. sculptum from the present study seems to belong to Babesia sensu stricto lineage, which is characterized by infecting erythrocytes from different vertebrate species and transovarial and transstadial transmission in ticks hosts (Schreeg et al., 2016). On the other hand, the sequences closely related to Theileria sp. and C. felis, obtained from $A$. sculptum nymphs and ocelots seems to belong to Theileria sp. and Cytauxzoon sp. lineage. Protozoans belonging to this lineage have developed strategies to enhance their propagation, which has been evidenced by enlarged schizont-infected leukocytes, suggesting a blocking of host cell apoptosis (Hagiwara et al., 1997; Susta et al., 2009). Piroplasmid sequences obtained from three coatis, one $T$. fosteri four A. parvum ticks seems to belong to T. equi lineage, in which host leukocyte manipulation has not been observed, differently from Theileria sp. and Cytauxzoon sp. lineage (Schreeg et al., 2016). In fact, the mitochondrial genome structure of $T$. equi has showed to be radically divergent from any other Piroplasmida species, suggesting that $T$. equi was recovered as a unique distinct lineage (Schreeg et al., 2016). Finally, although a genotype closely related to $R$. vitalii was detected in A. parvum ticks in the present study, the mitochondrial genome structure of $R$. vitalii has not been analysed yet, precluding its positioning in piroplasmid phylogroups. Schreeg et al. (2016) suggested that new lineages would be reported if a higher number of newly described piroplasmid were included in phylogenetic analyses. From a preliminary point of view, based on 18S rRNA and hsp70-concatenated phylogenetic analyses, $R$. vitalii and associated genotype detected in the present study grouped with protozoans allocated in Babesia s.s. group.

In conclusion, the present study revealed that wild animals and ticks in southern Pantanal region, Brazil, are exposed to a high diversity of piroplasmid species. The role of domestic dogs as a source of $B$. vogeli infection to wild animals should be better investigated. Wild and domestic animals and Amblyomma ticks seemed to be eventually infected by genotypes closely related to equine piroplasmid. Genotypes closely related to $R$. vitalii and ruminants-associated Theileria species circulate in Pantanal region. Finally, Cytauxzoon sp. was detected among freeliving wild felids for the first time in South America. Therefore, future studies are much needed in order to estimate the impact of piroplasmid infections among the Brazilian wildlife in Pantanal biome.

\section{Financial support}

We are thankful to Fundação de Amparo à Pesquisa do Estado de São Paulo (FAPESP) and Conselho Nacional de Desenvolvimento Científico e Tecnológico (CNPq) for the financial support to M.R. André (Process numbers \#2015/14896-1 and 473575/2014-0, respectively) and for Doctorate Scholarship to K. C. M Sousa (Process \#2013/131865) and the Scientific Initiation Fellowship to M. P. Fernandes (Process \#2016/10676-0).

\section{References}

Allsopp, M.T., Cavalier-Smith, T., De Waal, D.T., Allsopp, B.A., 1994. Phylogeny and evolution of the piroplasms. Parasitology 108 (Pt 2), 147-152.

Altschul, S.F., Gish, W., Miller, W., Myers, E.W., Lipman, D.J., 1990. Basic local alignment search tool. J. Mol. Biol. 215, 403-410.

Alvarado-Rybak, M., Solano-Gallego, L., Millán, J., 2016. A review of piroplasmid infections in wild carnivores worldwide: importance for domestic animal health and wildlife conservation. Parasit Vectors 9, 538.

André, M.R., Adania, C., Machado, R.Z., Allegretti, S., Felippe, P., Silva, K., Nakaghi, A. Dagnone, A., 2009. Molecular detection of Cytauxzoon spp. in asymptomatic Brazilian wild captive felids. J. Wildl. Dis. 45, 234-237.

André, M.R., Adania, C.H., Friciello, R.H., Allegretti, S., Machado, R.Z., 2011. Molecular and serological detection of Babesia spp. in Neotropical and exotic carnivores in Brazilian Zoos. J. Zoo Wildl. Med. 42, 139-143.

André, M.R., Denardi, N.C.B., Sousa, K.C.M., Gonçalves, L.R., Henrique, P.C., Ontivero, C.R.G.R., Gonzalez, I.H.L., Nery, C.V.C., Chagas, C.R.F., Monticelli, C., Santis, A.C.G.A., Machado, R.Z., 2014. Arthropod-borne pathogens circulating in freeroaming domestic cats in a zoo environment in Brazil. Ticks Tick Borne Dis. 5, 545-551.

André, M.R., Herrera, H.M., De Jesus Fernandes, S., De Sousa, K.C.M., Gonçalves, L.R. Domingos, I.H., De Macedo, G.C., Machado, R.Z., 2015. Tick-borne agents in domesticated and stray cats from the city of Campo Grande state of Mato Grosso do Sul, midwestern Brazil. Ticks Tick Borne Dis. 6, 779-786.

Battsetseg, B., Lucero, S., Xuan, X., Claveria, F.G., Inoue, N., Alhassan, A., Kanno, T., Igarashi, I., Nagasawa, H., Mikami, T., Fujisaki, K., 2002. Detection of natural infection of Boophilus microplus with Babesia equi and Babesia caballi in Brazilian horses using nested polymerase chain reaction. Vet. Parasitol. 107, 351-357.

Beck, R., Vojta, L., Mrljak, V., Marinculić, A., Beck, A., Zivicnjak, T., Cacciò, S.M., 2009. Diversity of Babesia and Theileria species in symptomatic and asymptomatic dogs in Croatia. Int. J. Parasitol. 39, 843-848.

Bell, T.G., Kramvis, A., 2013. Fragment merger: an online tool to merge overlapping long sequence fragments. Viruses 5, 824-833.

Benson, D.A., Mizrachi, I.K., Lipman, D.J., Ostell, J., Rapp, B.A., Wheeler, D.I., 2002. GenBank. Nucleic Acids Res. 30, 17-20.

Birkenheuer, A.J., Levy, M.G., Breitschwerdt, E.B., 2003. Development and evaluation of a seminested PCR for detection and differentiation of Babesia gibsoni (Asian genotype) and B. canis DNA in canine blood samples. J. Clin. Microbiol. 41, 4172-4177.

Birkenheuer, A.J., Marr, H., Alleman, A. R., Levy, M.G., Breitschwerdt, E. B., 2006. Development and evaluation of a PCR assay for the detection of Cytauxzoon felis DNA in feline blood samples. Vet. Parasitol. 137, 144-149.

Black, W.C., Piesman, J., 1994. Phylogeny of hard- and soft-tick taxa (Acari: Ixodida) based on mitochondrial 16S rDNA sequences. Proc. Natl. Acad. Sci. U. S. A. 91, 10034-10038.

Blouin, E.F., Kocan, A.A., Glenn, B.L., Kocan, K.M., Hair, J.A., 1984. Transmission of Cytauxzoon felis Kier, from bobcats, Felis rufus (Schreber), to domestic cats by Dermacentor variabilis (Say). J. Wildl. Dis. 20, 241-242.

Carli, E., Trotta, M., Chinelli, R., Drigo, M., Sinigoi, L., Tosolini, P., Furlanello, T., Millotti, A., Caldin, M., Solano-Gallego, L., 2012. Cytauxzoon sp. infection in the first endemic focus described in domestic cats in Europe. Vet. Parasitol. 183, 343-352.

Criado-Fornelio, A., Martinez-Marcos, A., Buling-Sarana, A., Barba-Carretero, J.C., 2003 Molecular studies on Babesia, Theileria and Hepatozoon in southern Europe. Part I. Epizootiological aspects. Vet. Parasitol. 113, 189-201.

Darriba, D., Taboada, G.L., Doalho, R., Posada, D., 2012. jModelTest 2: more models, new heuristics and parallel computing. Nat. Methods 9, 772.

Eiras, D.F., Craviotto, M.B., Baneth, G., Moré, G., 2014. First report of Rangelia vitalii infection (canine rangeliosis) in Argentina. Parasitol. Int. 63, 729-734.

Ewing, B., Green, P., 1998. Base-calling of automated sequencer traces using phred. II. Error probabilities. Genome Res. 8, 186-194.

Ewing, B., Hillier, L., Wendl, M.C., Green, P., 1998. Base-calling of automated sequencer traces using phred. I. Accuracy assessment. Genome Res. 8, 175-185.

Folmer, O., Black, M., Hoeh, W., Lutz, R., Vrijenhoek, R., 1994. DNA primers for amplification of mitochondrial cytochrome c oxidase subunit I from diverse metazoan invertebrates. Mol. Mar. Biol. Biotechnol. 3, 294-299.

Fredo, G., Bianchi, M.V., de Andrade, C.P., de Souza, S.O., Leite-Filho, R.V., Bandinelli, M.B., Amorim, D.B., Driemeier, D., Sonne, L., 2015. Natural infection of wild canids (Cerdocyon thous and Lycalopex gymnocercus) with the intraendothelial piroplasm Rangelia vitalii in southern Brazil. J. Wildl. Dis. 51, 880-884.

Fritz, D., 2010. A PCR study of piroplasms in 166 dogs and 111 horses in France (March 2006 to March 2008). Parasitol. Res. 106, 1339-1342.

Garner, M.M., Lung, M.P., Citino, S., Greiner, E.C., Harvey, J.W., Homer, B.L., 1996. Fatal cytauxzoonosis in a captive reared white tiger (Panthera tigris). Vet. Pathol. 33, $82-86$.

Gottlieb, J., André, M.R., Soares, J.F., Gonçalves, L.R., Tonial de Oliveira, M., Costa, M.M., Labruna, M.B., Bortolini, C.E., Machado, R.Z., Vieira, M.I., 2016. Rangelia vitalii, Babesia spp. and Ehrlichia spp. in dogs in Passo Fundo, state of Rio Grande do Sul, Brazil. Rev. Bras. Parasitol. Vet. 25, 172-178.

Haber, M.D., Tucker, M.D., Marr, H.S., Levy, J.K., Burgess, J., Lappin, M.R., Birkenheuer, A.J., 2007. The detection of Cytauxzoon felis in apparently healthy free-roaming cats in the USA. Vet. Parasitol. 146, 316-320. 
Hagiwara, K., Takahashi, K., Taniyama, H., Kawamoto, S., Kurosawa, T., Ikuta, K., Ishihara, C., 1997. Detection of Theileria sergenti schizonts in bovine lymph node. Int. J. Parasitol. 27, 1375-1378.

Hersh, M., Tibbetts, M., Strauss, M., Ostfeld, R., Keesing, F., 2012. Reservoir competence of wildlife host species for Babesia microti. Emerg. Infect. Dis. 18, 1951-1957.

Irwin, P.J., 2009. Canine babesiosis: from molecular taxonomy to control. Parasit Vectors. 2 (Suppl. 1), S4.

Jakob, W., Wesemeier, H.H., 1996. A fatal infection in a Bengal tiger resembling cytauxzoonosis in domestic cats. J. Comp. Pathol. 114, 439-444.

Jefferies, R., Ryan, U.M., Irwin, P.J., 2007. PCR-RFLP for the detection and differentiation of the canine piroplasm species and its use with filter paper-based technologies. Vet. Parasitol. 144, 20-27.

Katoh, K., Standley, D.M., 2013. MAFFT multiple sequence alignment software version 7: improvements in performance and usability. Mol. Biol. Evol. 30, 772-780.

Kjemtrup, A.M., Thomford, J., Robinson, T., Conrad, P.A., 2000. Phylogenetic relationships of human and wildlife piroplasm isolates in the western United States inferred from the 18 S nuclear small subunit RNA gene. Parasitology 120, 487-493.

Kuttler, K.L., 1988. Canine babesiosis. In: Ristic, M. (Ed.), Babesiosis of Domestic Animals and Man. CRC Press, Boca Raton, Florida, USA, pp. 12-13.

Labruna, M.B., Pereira, M.C., 2001. Carrapato em cães no Brasil. Clinic Vet. 30, 24-31.

Maia, L.M., Cerqueira, A.M., De Barros, M.D., De Souza, A.M., Moreira, N.S., Da Silva, A.V., Messick, J.B., Ferreira, R.F., Almosny, N.R., 2013. Cytauxzoon felis and 'Candidatus Mycoplasma haemominutum' coinfection in a Brazilian domestic cat (Felis catus). Rev. Bras. Parasitol. Vet. 22, 289-291.

Mamede, S.B., Alho, C.J.R., 2006. Response of wild mammals to seasonal shrinking-andexpansion of habitats due to flooding regime of the Pantanal, Brazil. Braz. J. Biol. 66, 991-998.

Meinkoth, J.H., Kocan, A.A., 2005. Feline cytauxzoonosis. Vet. Clin. North Am. Small Anim. Pract. 35, 89-101.

Melo, A.L., Witter, R., Martins, T.F., Pacheco, T.A., Alves, A.S., Chitarra, C.S., Dutra, V., Nakazato, L., Pacheco, R.C., Labruna, M.B., Aguiar, D.M., 2016. A survey of tickborne pathogens in dogs and their ticks in the Pantanal biome, Brazil. Med. Vet. Entomol. 30, 112-116.

Miller, M.A., Pfeiffer, W., Schwartz, T., 2010. Creating the CIPRES Science Gateway for inference of large phylogenetic trees. In: Proceedings of the Gateway Computing Environments Workshop (GCE). San Diego Supercomputer Center 9500 Gilman Drive La Jolla CA, USA. pp. 1-8.

Moraes, P.H., Rufino, C.P., Baraúna, A.R., Reis, T., Agnol, L.T., Meneses, A.M., Aguiar, D.C., Nunes, M.R., Gonçalves, E.C., 2015. Molecular characterization of Babesia vogeli in dogs from Belém, northern Brazil. Genet. Mol. Res. 14, 16364-16371.

Morrison, D.A., Ellis, J.T., 1997. Effects of nucleotide sequence alignment on phylogeny estimation: a case study of $18 \mathrm{~S}$ rDNAs of apicomplexa. Mol. Biol. Evol. 14, 428-441.

Nietfield, J.C., Pollock, C., 2002. Fatal cytauxzoonosis in a free-ranging bobcat (Lynx rufus). J. Wildl. Dis. 38, 607-610.

O’Dwyer, L.H., Lopes, V.V., Rubini, A.S., Paduan, K.S., Ribolla, P.E., 2009. Babesia spp. infection in dogs from rural areas of São Paulo State, Brazil. Rev. Bras. Parasitol. Vet. 18, 23-26.

Otiende, M.Y., Kivata, M.W., Makumi, J.N., Mutinda, M.N., Okun, D., Kariuki, L., Obanda, V., Gakuya, F., Mijele, D., Soriguer, R.C., Alasaad, S., 2015. Epidemiology of Theileria bicornis among black and white rhinoceros metapopulation in Kenya. BMC Vet. Res. 11.

Passos, L.M.F., Geiger, S.M., Ribeiro, M.F.B., Pfister, K., Zahler-Rinder, M., 2005. First molecular detection of Babesia vogeli in dogs from Brazil. Vet. Parasitol. 127, 81-85.

Peixoto, P.V., Soares, C.O., Scofield, A., Santiago, C.D., França, T., Barros, S.S., 2007. Fatal cytauxzoonosis in captive-reared lions in Brazil. Vet. Parasitol. 145, 383-387.

Penzhorn, B., 2006. Babesiosis of wild carnivores and ungulates. Vet. Parasitol. 138, $11-21$.

Posada, D., Buckley, T.R., 2004. Model selection and model averaging in phylogenetics: advantages of akaike information criterion and Bayesian approaches over likelihood ratio tests. Syst. Biol. 53, 793-808.

Quadros, R.M., Soares, J.F., Xavier, J.S., Pilati, C., da Costa, J.L., Miotto, B.A., Miletti, L.C., Labruna, M.B., 2015. Natural infection of the Wild Canid Lycalopex gymnocercus by the Protozoan Rangelia vitalii, the Agent of Canine Rangeliosis. J. Wildl. Dis. 51, 787-789.

Reichard, M.V., Edwards, A.C., Meinkoth, J.H., Snider, T.A., Meinkoth, K.R., Heinz, R.E., Little, S.E., 2010. Confirmation of Amblyomma americanum (Acari: Ixodidae) as a vector for Cytauxzoon felis (Piroplasmorida: Theileridae) to domestic cats. J. Med. Entomol. 47, 890-896.

Ribeiro, M.F., da Silveira, J.A., Bastos, C.V., 2011. Failure of the Amblyomma cajennense nymph to become infected by Theileria equi after feeding on acute or chronically infected horses. Exp. Parasitol. 128, 324-377.

Roby, T.O., Anthony, D.W., 1963. Transmission of equine piroplasmosis by the tropical tick Dermacentor nitens (Neumann). J. Am. Vet. Med. Assoc. 142, 768-769.

Ronquist, F., Huelsenbeck, J.P., 2003. MrBayes 3: Bayesian phylogenetic inference under mixed models. Bioinformatics 19, 1572-1574.

Sanger, F., Nicklen, S., Coulson, A.R., 1977. DNA sequencing with chain-terminating inhibitors. Proc. Natl. Acad. Sci. U. S. A. 74, 5463-5467.

Schnittger, L., Rodriguez, A., Florin-Christensen, M., Morrison, D., 2012. Babesia: a world emerging. Infect. Genet. Evol. 12, 1788-17809.

Schreeg, M.E., Marr, H.S., Tarigo, J.L., Cohn, L.A., Bird, D.M., Scholl, E.H., Levy, M.G., Wiegmann, B.M., Birkenheuer, A.J., 2016. Mitochondrial genome sequences and structures aid in the resolution of piroplasmida phylogeny. PLoS One 11, e0165702.

Scoles, G.A., Ueti, M.W., 2013. Amblyomma cajennense is an intrastadial biological vector of Theileria equi. Parasit Vectors 6, 306.

Silveira, J.A., Rabelo, E.M., Lacerda, A.C., Borges, P.A., Tomás, W.M., Pellegrin, A.O., Tomich, R.G., Ribeiro, M.F., 2013. Molecular detection and identification of hemoparasites in pampas deer (Ozotoceros bezoarticus Linnaeus, 1758) from the Pantanal Brazil. Ticks Tick Borne Dis. 4, 341-345.

Silveira, J.A., D'Elia, M.L., de Oliveira Avelar, I., de Almeida, L.R., Dos Santos, H.A., de Magalhães Soares, D.F., Ribeiro, M.F., Dos Santos Lima, W., Ecco, R., 2016. Rangelia vitalii in a free-ranging maned wolf (Chrysocyon brachyurus) and co-infections. Int. J. Parasitol. Parasites Wildl. 5, 280-285.

Soares, J.F., Girotto, A., Brandão, P.E., Da Silva, A.S., França, R.T., Lopes, S.T., Labruna, M.B., 2011. Detection and molecular characterization of a canine piroplasm from Brazil. Vet. Parasitol. 180, 203-208.

Soares, J.F., Dall'Agnol, B., Costa, F.B., Krawczak, F.S., Comerlato, A.T., Rossato, B.C., Linck, C.M., Sigahi, E.K., Teixeira, R.H., Sonne, L., Hagiwara, M.K., Gregori, F., Vieira, M.I., Martins, J.R., Reck, J., Labruna, M.B., 2014. Natural infection of the wild canid, Cerdocyon thous, with the piroplasmid Rangelia vitalii in Brazil. Vet. Parasitol. 202, 156-163.

Soares, J.F., Carvalho, L., Maya, L., Dutra, F., Venzal, J.M., Labruna, M.B., 2015. Molecular detection of Rangelia vitalii in domestic dogs from Uruguay. Vet. Parasitol. 210, 98-101.

Sousa, K.C.M., André, M.R., Herrera, H.M., De Andrade, G.B., Jusi, M.M., Dos Santos, L.L., Barreto, W.T., Machado, R.Z., De Oliveira, G.P., 2013. Molecular and serological detection of tick-borne pathogens in dogs from an area endemic for Leishmania in fantum in Mato Grosso do Sul, Brazil. Rev. Bras. Parasitol. Vet. 22, 525-531.

Sousa, K.C.M., Fernandes, M.P., Herrera, H.M., Benevenute, J.L., Santos, F.M., Rocha, F.L., Barreto, W.T.G., Macedo, G.C., Campos, J.B., Martins, T.F., Pinto, P.C.E.A., Barros-Battesti, D., Piranda, E.M., Cançado, P.H.D., Machado, R.Z., André, M.R., 2017a. Molecular detection of Hepatozoon spp. in domestic dogs and wild mammals in southern Pantanal, Brazil with implications in the transmission route. Vet. Parasitol. 237, 37-46.

Sousa, K.C.M., Herrera, H.M., Secato, C.T., Oliveira, A.D.V., Santos, F.M., Rocha, F.L., Barreto, W.T.G., Macedo, G.C., de Andrade Pinto, P.C.E., Machado, R.Z., Costa, M.T., André, M.R., 2017b. Occurrence and molecular characterization of hemoplasmas in domestic dogs and wild mammals in a Brazilian wetland. Acta Trop. 171, 172-181.

Stover, B.C., Muller, K.F., 2010. TreeGraph 2: combining and visualizing evidence from different phylogenetic analyses. BMC Bioinf. 11, 1-9.

Susta, L., Torres-Velez, F., Zhang, J., Brown, C., 2009. An in situ hybridization and immunohistochemical study of cytauxzoonosis in domestic cats. Vet. Pathol. 46, 1197-1204.

Ujvari, B., Madsen, T., Olsson, M., 2004. High prevalence of Hepatozoon spp. (Apicomplexa, Hepatozoidae) infection in water pythons (Liasis fuscus) from tropical Australia. J. Parasitol. 90, 670-672.

Wolf, R.W., Aragona, M., Muñoz-Leal, S., Pinto, L.B., Melo, A.L.T., Braga, I.A., Costa, J.D.S., Martins, T.F., Marcili, A., Pacheco, R.D.C., Labruna, M.B., Aguiar, D.M., 2016. Novel Babesia and Hepatozoon agents infecting non-volant small mammals in the Brazilian Pantanal, with the first record of the tick Ornithodoros guaporensis in Brazil. Ticks Tick Borne Dis. 7, 449-456.

Yabsley, M., Shock, B., 2013. Natural history of Zoonotic Babesia: role of wildlife reservoirs. Int. J. Parasitol. Parasites Wildl. 2, 18-31.

Yabsley, M.J., Murphy, S.M., Cunningham, M.W., 2006. Molecular detection and characterization of Cytauxzoon felis and a Babesia species in cougars from Florida. J. Wildl. Dis. $42,366-374$.

da Costa, A.P., Costa, F.B., Labruna, M.B., Silveira, I., Moraes-Filho, J., Soares, J.F., Spolidorio, M.G., Guerra, R.M., 2015. A serological and molecular survey of Babesia vogeli, Ehrlichia canis and Rickettsia spp. among dogs in the state of Maranhão, northeastern Brazil. Rev. Bras. Parasitol. Vet. 24, 28-35. 